\title{
An Efficient Series Solution for Nonlinear Multiterm Fractional Differential Equations
}

\author{
Moh'd Khier Al-Srihin and Mohammed Al-Refai \\ Department of Mathematical Sciences, United Arab Emirates University, Al-Ain, UAE \\ Correspondence should be addressed to Mohammed Al-Refai; m_alrefai@uaeu.ac.ae
}

Received 24 January 2017; Accepted 21 February 2017; Published 8 March 2017

Academic Editor: Thabet Abdeljawad

Copyright (C) 2017 Moh'd Khier Al-Srihin and Mohammed Al-Refai. This is an open access article distributed under the Creative Commons Attribution License, which permits unrestricted use, distribution, and reproduction in any medium, provided the original work is properly cited.

\begin{abstract}
In this paper, we introduce an efficient series solution for a class of nonlinear multiterm fractional differential equations of Caputo type. The approach is a generalization to our recent work for single fractional differential equations. We extend the idea of the Taylor series expansion method to multiterm fractional differential equations, where we overcome the difficulty of computing iterated fractional derivatives, which are difficult to be computed in general. The terms of the series are obtained sequentially using a closed formula, where only integer derivatives have to be computed. Several examples are presented to illustrate the efficiency of the new approach and comparison with the Adomian decomposition method is performed.
\end{abstract}

\section{Introduction}

Fractional differential equations (FDEs) are generalization to differential equations (DEs) for noninteger orders. In recent years, FDEs caught the attention of many researchers because of their appearance in modeling several phenomenon in the physical sciences. As many FDEs do not possess exact solutions on closed forms, analytical and numerical techniques have been implemented to study these equations. Iterative methods, such as the variational iteration method (VIM) in [1], the homotopy analysis method (HAM) in [2,3], the Adomian decomposition method (ADM) in [4-9], and the fractional differential transform method in [10], have been implemented to solve various types of FDEs. These methods produce a solution in a series form whose terms are determined sequentially. We refer the reader to $[11,12]$ for a comprehensive study of series solutions of fractional differential equations. Recently, we have introduced a new series solution for single fractional differential equations [13]. The new approach is a modified form of the well-known Taylor series expansion, where we overcome the difficulty of computing iterative fractional derivatives. The efficiency of the new approach has been illustrated through several examples. In this paper we extend the idea to multiterm fractional differential equations. The presented work is a part of the Master thesis [14]. We consider the left Caputo fractional derivative $D_{0^{+}}^{\alpha}$, defined by $[15,16]$

$$
\begin{aligned}
& D_{0^{+}}^{\alpha} f(t) \\
& = \begin{cases}\frac{1}{\Gamma(n-\alpha)} \int_{0}^{t}(t-\tau)^{n-\alpha-1} f^{(n)}(\tau) d \tau, & \text { if } n-1<\alpha<n, \\
f^{(n)}(t), & \text { if } \alpha=n \in \mathbb{N}\end{cases}
\end{aligned}
$$

provided the integral exists, where $\Gamma$ is the well-known Gamma function. The left Riemann-Liouville fractional integral, $I_{0^{+}}^{\alpha}$, of order $\alpha \in \mathbb{R}^{+}$, is defined by

$$
I_{0^{+}}^{\alpha} f(t)=\frac{1}{\Gamma(\alpha)} \int_{0}^{t}(t-s)^{\alpha-1} f(s) d s, \quad t>0, \alpha>0 .
$$

The left Caputo derivative is related to the left RiemannLiouville fractional integral by

$$
D_{0^{+}}^{\alpha} f(t)=I_{0^{+}}^{n-\alpha} f^{(n)}(t), \quad n-1<\alpha<n .
$$


It is known that

$$
\begin{aligned}
& \left(\left(I_{0^{+}}^{\alpha} D_{0^{+}}^{\alpha}\right) f\right)(t)=f(t)-\sum_{k=0}^{m-1} \frac{f^{(k)}(0)}{k !} t^{k}, \\
& \left(\left(D_{0^{+}}^{\alpha} I_{0^{+}}^{\alpha}\right) f\right)(t)=f(t) .
\end{aligned}
$$

Also, for $n-1<\alpha<n, n \in \mathbb{N}, t>0$, it holds that

$$
\begin{aligned}
& D_{0^{+}}^{\alpha}\left(t^{\mu}\right) \\
& \quad= \begin{cases}\frac{\Gamma(\mu+1)}{\Gamma(\mu-\alpha+1)} t^{\mu-\alpha}, & \text { if } \mu>n-1, \\
0, & \text { if } \mu \in\{0,1, \ldots, n-1\} .\end{cases}
\end{aligned}
$$

This paper is organized as follows. In Section 2, we present the series solution of nonlinear two-term fractional differential equations. We illustrate the efficiency of the presented technique through several examples. We also compare our results with the ones obtained by the Adomian decomposition method. In Section 3, we present and illustrate the efficiency of the new series solution for three-term fractional differential equations of several types. Finally, we conclude with some remarks in Section 4.

\section{Two-Term Fractional Differential Equations}

We start with the nonlinear two-term fractional initial value problems of the form

$$
c_{1} D_{0^{+}}^{\alpha_{1}} u(t)+c_{2} D_{0^{+}}^{\alpha_{2}} u(t)=f(t, u(t)), \quad t>0
$$

with

$$
u(0)=b
$$

where $0<\alpha_{2} \leq \alpha_{1}<1$, and $c_{1}$ and $c_{2}$ are nonzero constants. We assume that $f(t, u(t))$ is continuous and smooth with respect to $u(t)$. We also assume that $\alpha_{1}$ and $\alpha_{2}$ are rational numbers with $\alpha_{1}=p_{1} / q_{1}$ and $\alpha_{2}=p_{2} / q_{2}, p_{1}, p_{2}, q_{1}, q_{2} \in \mathbb{N}$.

2.1. The Expansion Procedure. Let $q=\operatorname{lcm}\left(q_{1}, q_{2}\right)$; we have $q=s q_{1}=r q_{2}$ for some $s, r \in \mathbb{N}$.

In the following we expand the solution of problem (6)(7) in an infinite series of the form

$$
u(t)=\sum_{n=0}^{\infty} a_{n} t^{n / q},
$$

where the coefficients $a_{n}: n \geqslant 0$ have to be determined sequentially in the following manner: From the initial condition (7) we have $u(0)=b=a_{0}$. Since $D_{0^{+}}^{\alpha}(c)=0$, for $c$ being constant, we have

$$
\begin{aligned}
& D_{0^{+}}^{\alpha_{1}} u(t)=\sum_{n=1}^{\infty} a_{n} s_{n} t^{n / q-p_{1} / q_{1}}=\sum_{n=1}^{\infty} a_{n} s_{n} t^{\left(n-s p_{1}\right) / q}, \\
& D_{0^{+}}^{\alpha_{2}} u(t)=\sum_{n=1}^{\infty} a_{n} r_{n} t^{n / q-p_{2} / q_{2}}=\sum_{n=1}^{\infty} a_{n} r_{n} t^{\left(n-r p_{2}\right) / q},
\end{aligned}
$$

where

$$
\begin{aligned}
& s_{n}=\frac{\Gamma(n / q+1)}{\Gamma\left(n / q-\alpha_{1}+1\right)}, \\
& r_{n}=\frac{\Gamma(n / q+1)}{\Gamma\left(n / q-\alpha_{2}+1\right)} .
\end{aligned}
$$

By substituting (9) in (6) we have

$$
\begin{aligned}
& c_{1} \sum_{n=1}^{\infty} a_{n} s_{n} t^{\left(n-s p_{1}\right) / q}+c_{2} \sum_{n=1}^{\infty} a_{n} r_{n} t^{\left(n-r p_{2}\right) / q} \\
& \quad=f\left(t, \sum_{n=0}^{\infty} a_{n} t^{n / q}\right) .
\end{aligned}
$$

Applying the well-known Taylor series method to compute the coefficients $\left\{a_{n} ; n \geq 1\right\}$ will lead to computing iterated fractional derivatives, which are not easily computed in general. To avoid this difficulty, let $t=w^{q}$; we have

$$
\begin{gathered}
c_{1} \sum_{n=1}^{\infty} a_{n} s_{n} w^{n-s p_{1}}+c_{2} \sum_{n=1}^{\infty} a_{n} r_{n} w^{n-r p_{2}} \\
\quad=f\left(w^{q}, \sum_{n=0}^{\infty} a_{n} w^{n}\right) .
\end{gathered}
$$

Shifting the index to zero yields

$$
\begin{aligned}
& c_{1} \sum_{n=0}^{\infty} a_{n+1} s_{n+1} w^{n-s p_{1}+1}+c_{2} \sum_{n=0}^{\infty} a_{n+1} r_{n+1} w^{n-r p_{2}+1} \\
& \quad=f\left(w^{q}, \sum_{n=0}^{\infty} a_{n} w^{n}\right) .
\end{aligned}
$$

To avoid the singularity at $w=0$, we multiply (13) by $w^{s p_{1}-1}$; we have

$$
\begin{aligned}
& c_{1} \sum_{n=0}^{\infty} a_{n+1} s_{n+1} w^{n}+c_{2} \sum_{n=0}^{\infty} a_{n+1} r_{n+1} w^{n-r p_{2}+s p_{1}} \\
& \quad=w^{s p_{1}-1} f\left(w^{q}, \sum_{n=0}^{\infty} a_{n} w^{n}\right) .
\end{aligned}
$$

Now, since $\alpha_{1}=p_{1} / q_{1}=s p_{1} / q>r p_{2} / q=p_{2} / q_{2}=\alpha_{2}$, thus $s p_{1}-r p_{2}>0$, and (14) has no singularity at $w=0$.

Let $k=s p_{1}-r p_{2}-1 \geq 0$, and $g(w)=f\left(w^{q}, \sum_{n=0}^{\infty} a_{n} w^{n}\right)$; then (14) can be written as

$$
\begin{aligned}
& c_{1} \sum_{n=0}^{k} a_{n+1} s_{n+1} w^{n}+\sum_{n=k+1}^{\infty}\left(c_{1} a_{n+1} s_{n+1}+c_{2} a_{n-k} r_{n-k}\right) w^{n} \\
& \quad=w^{k+r p_{2}} g(w) .
\end{aligned}
$$

We first determine the coefficients $a_{n}$ for $n \leq k$. By performing the $n$th derivativeof (15) with respect to $w$ and 
substituting $w=0$, we have

$$
c_{1} n ! a_{n+1} s_{n+1}=\left.\frac{\mathrm{d}^{n}}{\mathrm{~d} w^{n}}\left(w^{k+r p_{2}} g(w)\right)\right|_{w=0}
$$

which yields

$$
a_{n+1}=\left.\frac{1}{c_{1} n ! s_{n+1}} \frac{\mathrm{d}^{n}}{\mathrm{~d} w^{n}}\left(w^{k+r p_{2}} f\left(w^{q}, \sum_{n=0}^{\infty} a_{n} w^{n}\right)\right)\right|_{w=0} .
$$

Since $k+r p_{2} \geq n+1$, and $f$ is smooth, then for $n \leq k$, we have

$$
\left.\frac{\mathrm{d}^{n}}{\mathrm{~d} w^{n}}\left(w^{k+r p_{2}} f\left(w^{q}, \sum_{n=0}^{\infty} a_{n} w^{n}\right)\right)\right|_{w=0}=0
$$

and hence $a_{n+1}=0$, for $n \leq k$.

We now determine $a_{n}$, for $n \geq k+1$. By performing the $n$th derivative of (15) with respect to $w$ and substituting $w=0$, we have

$$
\begin{gathered}
n !\left(c_{1} a_{n+1} s_{n+1}+c_{2} a_{n-k} r_{n-k}\right)=\left.\frac{\mathrm{d}^{n}}{\mathrm{~d} w^{n}}\left(w^{k+r p_{2}} g(w)\right)\right|_{w=0} \\
=\left.\frac{\mathrm{d}^{n}}{\mathrm{~d} w^{n}}\left(w^{k+r p_{2}} f\left(w^{q}, \sum_{n=0}^{\infty} a_{n} w^{n}\right)\right)\right|_{w=0} .
\end{gathered}
$$

Using the well-known Leibniz rule for differentiating the products, we have

$$
\begin{aligned}
& \frac{\mathrm{d}^{n}}{\mathrm{~d} w^{n}}\left(w^{k+r p_{2}} g(w)\right) \\
& \quad=\sum_{j=0}^{n}\left(\begin{array}{l}
n \\
j
\end{array}\right) \frac{\mathrm{d}^{j}}{\mathrm{~d} w^{j}}\left(w^{k+r p_{2}}\right) \frac{\mathrm{d}^{n-j}}{\mathrm{~d} w^{n-j}}(g(w)) .
\end{aligned}
$$

Since

$$
\left.\frac{\mathrm{d}^{j}}{\mathrm{~d} w^{j}}\left(w^{k+r p_{2}}\right)\right|_{w=0}= \begin{cases}0 & \text { if } j \neq k+r p_{2} \\ j ! & \text { if } j=k+r p_{2}\end{cases}
$$

we have

$$
\begin{aligned}
& c_{1} a_{n+1} s_{n+1}+c_{2} a_{n-k} r_{n-k} \\
& =\left.\frac{1}{(n-j) !}\left(\frac{\mathrm{d}^{n-j}}{\mathrm{~d} w^{n-j}} g(w)\right)\right|_{w=0}, \\
& \text { where } j=k+r p_{2} .
\end{aligned}
$$

From the last equation we determine $a_{n}: n \geq k+1$ and thus the solution

$$
u(t)=\sum_{n=0}^{\infty} a_{n} t^{n / q}=a_{0}+\sum_{n=k+1}^{\infty} a_{n} t^{n / q}
$$

is obtained.
Remark 1. In (6), assuming $\alpha_{1}=\alpha_{2}=1$, then $s_{n}=r_{n}=n, k=$ -1 and $k+r p_{2}=0$. Thus (17) is reduced to

$$
a_{n+1}=\frac{1}{c_{1}(n+1) !} \frac{\mathrm{d}^{n}}{\mathrm{~d} w^{n}}\left[f\left(w, \sum_{n=0}^{\infty} a_{n} w^{n}\right)\right]_{w=0},
$$

which coincides with the coefficients obtained by the Taylor series expansion method. Same comment applies for the coefficient $a_{n+1}$ in (22).

Remark 2. The algorithm can be generalized for the two-term fractional differential equation (6) with $0<\alpha_{2} \leq \alpha_{1}<N$, for arbitrary integer $N$. But we have to take care of the existence of the fractional derivative $D_{0^{+}}^{\alpha_{1}}\left(t^{n / q}\right)$ (see Eq. (5)), by choosing the coefficient $a_{n}=0$, for $n=1, \ldots, q N-1$. This case is discussed in Section 3.

\subsection{Numerical Results}

Example 3. Consider the nonlinear two-term fractional initial value problem

$$
\begin{aligned}
& 2 D_{0^{+}}^{1 / 2} u(t)+2 \Gamma\left(\frac{13}{10}\right) D_{0^{+}}^{1 / 5} u(t) \\
& \quad=\Gamma\left(\frac{1}{2}\right)\left(u^{2}+t^{3 / 10}-t+1\right), \quad t>0,
\end{aligned}
$$

with

$$
u(0)=0
$$

The exact solution of the problem is $u(t)=t^{1 / 2}$.

Applying the current algorithm, we have $\alpha_{1}=1 / 2=$ $p_{1} / q_{1}, \alpha_{2}=1 / 5=p_{2} / q_{2}, q=1 \cdot \operatorname{com}\left(q_{1}, q_{2}\right)=10, s=5$, and $r=2$. We expand the solution in an infinite series of the form

$$
u(t)=\sum_{n=0}^{\infty} a_{n} t^{n / 10}
$$

The initial condition in (26) yields $a_{0}=0$. We have $t=w^{q}=$ $w^{10}$ and

$$
\begin{aligned}
g(w) & =f\left(w^{10}, u(w)\right) \\
& =\Gamma\left(\frac{1}{2}\right)\left(\left(\sum_{n=0}^{\infty} a_{n} w^{n}\right)^{2}+w^{3}-w^{10}+1\right) .
\end{aligned}
$$

Since $g(w)$ is continuous and smooth with respect to $w$, we have

$$
a_{n+1}=0, \quad \text { for } n \leq k=s p_{1}-r p_{2}-1=2 \text {. }
$$

Thus $a_{1}=a_{2}=a_{3}=0$. The function $g(w)$ satisfies the assumption of the proposed algorithm, and it holds that 


$$
\left.\frac{\mathrm{d}^{m}}{\mathrm{~d} w^{m}}(g(w))\right|_{w=0}=\Gamma\left(\frac{1}{2}\right) \begin{cases}1 & m=0 \\ 3 ! & m=3 \\ 6 ! a_{3}^{2} & m=6 \\ 2 \times 7 ! a_{3} a_{4} & m=7 \\ 8 ! a_{4}^{2}+2 \times 8 ! a_{3} a_{5} & m=8 \\ 2 !\left(a_{4} a_{5}+a_{3} a_{3}\right) & m=9 \\ -10 !+10 ! a_{5}^{2}+2 \times 10 !\left(a_{4} a_{6}+a_{3} a_{7}\right) & m=10 \\ \vdots & \vdots\end{cases}
$$

The computation above is made using the software Mathematica version 9. For $n \geq 3$; substituting (30) in (22) yields

$$
\begin{aligned}
& 2 a_{n+1} s_{n+1}+2 \Gamma\left(\frac{13}{10}\right) a_{n-2} r_{n-2} \\
& =\left.\frac{1}{(n-4) !} \frac{\mathrm{d}^{n-4}}{\mathrm{~d} w^{n-4}}(g(w))\right|_{w=0},
\end{aligned}
$$

where

$$
\begin{aligned}
& s_{n+1}=\frac{\Gamma((n+1) / 10+1)}{\Gamma((n+1) / 10+1 / 2)}, \\
& r_{n-2}=\frac{\Gamma((n-2) / 10+1)}{\Gamma((n-2) / 10+4 / 5)} .
\end{aligned}
$$

Applying (31) together with $a_{1}=a_{2}=a_{3}=0$, we have

$$
\begin{aligned}
& a_{4}=0, \\
& a_{5}=1, \\
& a_{n}=0, \\
& n \geq 6 .
\end{aligned}
$$

Thus,

$$
u(t)=a_{5} t^{5 / 10}=t^{1 / 2}
$$

and the exact solution of problem (25)-(26) is obtained.

In the following we compare our results with the Adomian decomposition method (ADM). Assume that the nonlinear function $f(u)=u^{2}$ and the solution $u(t)$ can be expressed in the following series form:

$$
\begin{aligned}
& u(t)=\sum_{n=0}^{\infty} u_{n}(t), \\
& f(u)=\sum_{n=0}^{\infty} A_{n},
\end{aligned}
$$

where $A_{n}, n=0,1,2, \ldots$ are the well-known Adomian polynomials that can be derived from [17]

$$
A_{n}=\frac{1}{n !} \frac{\mathrm{d}^{n}}{\mathrm{~d} \beta^{n}}\left[f\left(\sum_{j=0}^{\infty} \beta^{j} u_{j}\right)\right]_{\beta=0}, \quad j \geq 0 .
$$

For recent advances in the Adomian decomposition method we refer the reader to $[18,19]$. For $f(u)=u^{2}$, we have

$$
\begin{gathered}
A_{0}=u_{0}^{2}, \\
A_{1}=2 u_{0} u_{1}, \\
A_{2}=2 u_{0} u_{2}+u_{1}^{2}, \\
A_{3}=2 u_{0} u_{3}+2 u_{1} u_{2}, \\
A_{4}=2 u_{0} u_{4}+2 u_{1} u_{3}+u_{2}^{2},
\end{gathered}
$$

Applying the Riemann-Liouville fractional integral operator $I_{0^{+}}^{1 / 2}$ to (25) and substituting

$$
I_{0^{+}}^{1 / 2} D_{0^{+}}^{1 / 5} u(t)=I_{0^{+}}^{3 / 10} u(t)
$$

we have

$$
\begin{aligned}
u(t)= & \frac{\Gamma(1 / 2)}{2} I_{0^{+}}^{1 / 2}\left(1+t^{3 / 10}-t\right)-\Gamma\left(\frac{13}{10}\right) I_{0^{+}}^{3 / 10} u(t) \\
& +\frac{\Gamma(1 / 2)}{2} I_{0^{+}}^{1 / 2}\left(u^{2}(t)\right) \\
= & t^{1 / 2}+0.853958 t^{4 / 5}-0.666667 t^{3 / 2} \\
& -\Gamma\left(\frac{13}{10}\right) I_{0^{+}}^{3 / 10}\left(\sum_{n=0}^{\infty} u_{n}(t)\right) \\
& +\frac{\Gamma(1 / 2)}{2} I_{0^{+}}^{1 / 2}\left(\sum_{n=0}^{\infty} A_{n}\right)=\sum_{n=0}^{\infty} u_{n}(t)
\end{aligned}
$$


Set

$$
u_{0}=t^{1 / 2}+0.853958 t^{4 / 5}-0.666667 t^{3 / 2}
$$

and balancing (39) yields

$$
\begin{aligned}
u_{n+1}=-\Gamma\left(\frac{13}{10}\right) I_{0^{+}}^{3 / 10}\left(u_{n}\right)+\frac{\Gamma(1 / 2)}{2} I_{0^{+}}^{1 / 2}\left(A_{n}\right), & \\
n & =0,1,2, \ldots
\end{aligned}
$$

Evaluating the first 5 term of the power series solution, we obtain the approximate solution $u^{(4)}(t)=\sum_{n=0}^{4} u_{n}(t)$, where

$$
\begin{aligned}
u^{(4)}(t)= & t^{0.5}+0.257998 t^{2}-2.798359 t^{2.7} \\
& -1.454522 t^{3}+8.855605 t^{3.4}+9.799202 t^{3.7} \\
& +2.317936 t^{4}-11.599906 t^{4.1} \\
& -22.429449 t^{4.4}-11.273123 t^{4.7} \\
& +6.685081 t^{4.8}-1.388180 t^{5} \\
& +23.109057 t^{5.1}+20.278401 t^{5.4} \\
& -1.404129 t^{5.5}+5.305238 t^{5.7} \\
& -11.070905 t^{5.8}+0.278605 t^{6} \\
& -17.334122 t^{6.1}-7.918245 t^{6.4} \\
& +2.012699 t^{6.5}-0.885674 t^{6.7} \\
& +7.156236 t^{6.8}+5.827855 t^{7.1} \\
& +1.140180 t^{7.4}-1.153180 t^{7.5} \\
& -2.127835 t^{7.8}-0.742611 t^{8.1} \\
& +0.309488 t^{8.5}+0.244589 t^{8.8} \\
& -0.032578 t^{9.5}
\end{aligned}
$$

which is not the exact solution. Solving the problem by the $\mathrm{ADM}$ with $u_{0}=t^{0.5}$ yields $U^{(4)}(t)=\sum_{n=0}^{4} U_{n}(t)$, where

$$
\begin{aligned}
U^{(4)}(t)= & t^{0.5}-0.853958 t^{0.8}+0.682106 t^{1.1} \\
& -0.515733 t^{1.4}+0.666667 t^{1.5} \\
& +0.0372208 t^{1.7}-1.527774 t^{1.8} \\
& +2.25291 t^{2.1}-2.68363 t^{2.4}+0.711111 t^{2.5} \\
& -2.576649 t^{2.8}+5.473545 t^{3.1} \\
& +0.8533333 t^{3.5}-4.19714 t^{3.8} \\
& +1.078789 t^{4.5}
\end{aligned}
$$

which is again not the exact solution. Figure 1 depicts the exact solution $u(t)$ obtained by the current algorithm and

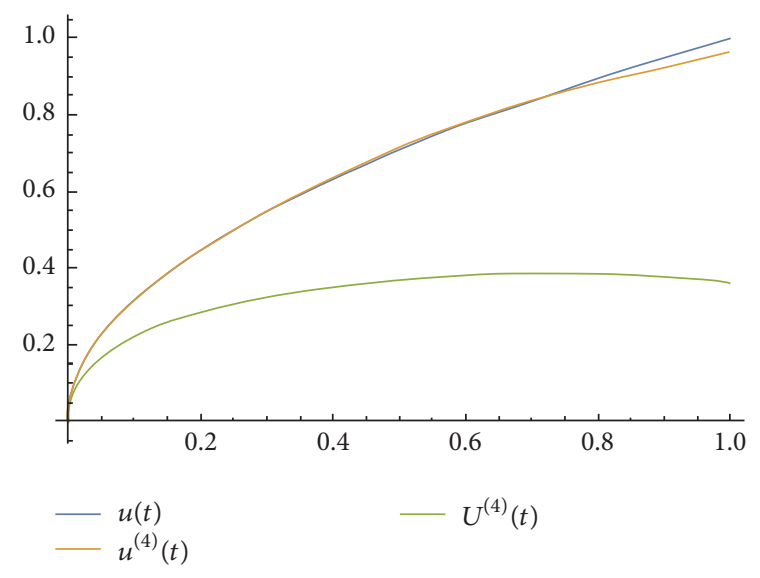

FIgURE 1: Comparison between the exact and approximate solutions obtained by the ADM for problem ((25)-(26)).

the approximate solutions $u^{(4)}(t)$ and $U^{(4)}(t)$ obtained by the Adomian decomposition method.

Example 4. Consider the nonlinear two-term fractional initial value problem

$$
D_{0^{+}}^{4 / 5} u(t)+D_{0^{+}}^{1 / 2} u(t)=\frac{u(t) \sin (t)}{u^{2}(t)+1}, \quad t>0,
$$

with

$$
u(0)=1
$$

This example has been discussed in [20], where the problem is transformed to a fractional integral equation, and then the Adams-Bashforth-Moulton method is used with step size $h=$ $1 / 50$ to approximate the solution.

Applying the current algorithm, we have $p_{1}=4, q_{1}=$ $5, p_{2}=1, q_{2}=2, q=1 \cdot \operatorname{c} \cdot \mathrm{m}(5,2)=10, s=2, r=5, k=2$, and

$$
\begin{aligned}
& a_{n+1} s_{n+1}+a_{n-2} r_{n-2} \\
& \quad=\left.\frac{1}{(n-7) !}\left(\frac{\mathrm{d}^{n-7}}{\mathrm{~d} w^{n-7}} f\left(w^{10}, \sum_{n=0}^{\infty} a_{n} w^{n}\right)\right)\right|_{w=0},
\end{aligned}
$$

where

$$
f\left(w^{10}, \sum_{n=0}^{\infty} a_{n} w^{n}\right)=\frac{\sin \left(w^{10}\right) \sum_{n=0}^{\infty} a_{n} w^{n}}{\left(\sum_{n=0}^{\infty} a_{n} w^{n}\right)^{2}+1} .
$$


TABLE 1: The error of Example 4 for $N=35$ and different values of $t$.

\begin{tabular}{lc}
\hline$t_{i}$ & $E_{35}\left(t_{i}\right)$ \\
\hline 0.1 & $1.16844 \times 10^{-10}$ \\
0.2 & $8.36081 \times 10^{-9}$ \\
0.3 & $9.93967 \times 10^{-8}$ \\
0.4 & $5.28261 \times 10^{-7}$ \\
0.5 & $1.93074 \times 10^{-6}$ \\
0.6 & $5.47664 \times 10^{-6}$ \\
0.7 & 0.0000130833 \\
0.8 & 0.0000276624 \\
0.9 & 0.0000534896 \\
1.0 & 0.0000967893 \\
\hline
\end{tabular}

The initial condition $u(0)=1$ yields $a_{0}=1$. We apply (46) to compute the first 13 nonzero terms of $a_{n}$, and we have

$$
\begin{aligned}
u(t)= & 1+0.298242 t^{1.8}-0.227519 t^{2.1} \\
& +0.167717 t^{2.4}-0.119885 t^{2.7}+0.0833333 t^{3} \\
& -0.0564631 t^{3.3}+0.0373656 t^{3.6} \\
& -0.0280303 t^{3.8}-0.0241927 t^{3.9} \\
& +0.0179008 t^{4.1}+0.0153477 t^{4.2} \\
& -0.0112111 t^{4.4}
\end{aligned}
$$

Since the exact solution of problem (44)-(45) is not available in a closed form, we define the error $E_{N}(t)$ by

$$
E_{N}\left(t_{i}\right)=\int_{0}^{t_{i}}\left(\mathscr{P} u_{N}(t)\right)^{2} \mathrm{~d} t, \quad t \in\left[0, t_{i}\right]
$$

where

$$
\begin{aligned}
& \mathscr{P} u(t)=D_{0^{+}}^{4 / 5} u(t)+D_{0^{+}}^{1 / 2} u(t)-\frac{u(t) \sin (t)}{u^{2}(t)+1}, \\
& u_{N}(t)=\sum_{k=0}^{N} a_{k} t^{k / 10} .
\end{aligned}
$$

Tables 1 and 2 present the error $E_{N}$ for different values of $N$. One can see that the error decreases with $N$ and more accuracy can be achieved by considering more terms. Also, the error increases with $t$, as any other series solutions.

\section{Three-Term Fractional Differential Equations}

We consider the nonlinear three-term fractional initial value problem of the form

$$
\begin{aligned}
& c_{1} D_{0^{+}}^{\alpha_{1}} u(t)+c_{2} D_{0^{+}}^{\alpha_{2}} u(t)+c_{3} D_{0^{+}}^{\alpha_{3}} u(t)=f(t, y(t)), \\
& t>0,
\end{aligned}
$$

TABLE 2: The error of Example 4 at $t=1$ and for different values of N.

\begin{tabular}{lc}
\hline$N$ & $E_{N}(1)$ \\
\hline 5 & 0.0681689 \\
10 & 0.0681689 \\
15 & 0.0681689 \\
20 & 0.067155 \\
25 & 0.0248829 \\
30 & 0.00882027 \\
35 & 0.0000967893 \\
\hline
\end{tabular}

with

$$
u^{(i)}(0)=b_{i}, \quad i=0,1, \ldots, N_{1}-1,
$$

where

$$
\begin{aligned}
& 0<\alpha_{3} \leq \alpha_{2} \leq \alpha_{1}<N_{1}, \\
& N_{1}-1<\alpha_{1}<N_{1}, \\
& N_{2}-1<\alpha_{2} \leq N_{2} \leq N_{1}, \\
& 0<N_{3}-1<\alpha_{3} \leq N_{3} \leq N_{2},
\end{aligned}
$$

and $c_{1}, c_{2}$, and $c_{3}$ are nonzero constants. We assume that $f(t, y(t))$ is continuous and smooth with respect to $u(t)$. We also assume that $\alpha_{1}, \alpha_{2}$, and $\alpha_{3}$ are rational numbers with $\alpha_{1}=p_{1} / q_{1}, \alpha_{2}=p_{2} / q_{2}$, and $\alpha_{3}=p_{3} / q_{3}$, where $p_{1}, p_{2}, p_{3}, q_{1}, q_{2}, q_{3} \in \mathbb{N}$. Let $q=\operatorname{lcm}\left(q_{1}, q_{2}, q_{3}\right)$; we have $q=s q_{1}=r q_{2}=v q_{3}$ for $s, r$ and $v \in \mathbb{N}$.

Applying analogous steps for the case of the two-term fractional equations, we seek a solution of the form

$$
\begin{aligned}
u(t) & =\sum_{n=0}^{\infty} a_{n} t^{n / q}=\sum_{n=0}^{\left(N_{1}-1\right) q} a_{n} t^{n / q}+\sum_{n=\left(N_{1}-1\right) q+1}^{\infty} a_{n} t^{n / q} \\
& =\sum_{n=0}^{N_{1}-1} a_{n q} t^{n}+\sum_{n=\left(N_{1}-1\right) q+1}^{\infty} a_{n} t^{n / q} .
\end{aligned}
$$

From the initial condition (52) we have $u^{(i)}(0)=b_{i}=i ! a_{i q}$, and thus, $a_{i q}=b_{i} / i$ !, $i=0,1, \ldots, N_{1}-1$.

Using the fact that $D_{0^{+}}^{\alpha}\left(t^{n}\right)=0$, for $\alpha>n \in \mathbb{N}$, we have

$$
\begin{aligned}
& D_{0^{+}}^{\alpha_{1}} u(t)=\sum_{n=\left(N_{1}-1\right) q+1}^{\infty} a_{n} s_{n} t^{\left(n-s p_{1}\right) / q}, \\
& D_{0^{+}}^{\alpha_{2}} u(t)=\sum_{n=N_{2}}^{N_{1}-1} a_{n q} \rho_{n q} t^{n-\alpha_{2}}+\sum_{n=\left(N_{1}-1\right) q+1}^{\infty} a_{n} r_{n} t^{\left(n-r p_{2}\right) / q}, \\
& D_{0^{+}}^{\alpha_{3}} u(t)=\sum_{n=N_{3}}^{N_{1}-1} a_{n q} \nu_{n q} t^{n-\alpha_{3}}+\sum_{n=\left(N_{1}-1\right) q+1}^{\infty} a_{n} v_{n} t^{\left(n-v p_{3}\right) / q},
\end{aligned}
$$

where $s_{n}=\Gamma(n / q+1) / \Gamma\left(n / q-\alpha_{1}+1\right), r_{n}=\Gamma(n / q+1) / \Gamma(n / q-$ $\left.\alpha_{2}+1\right), \rho_{n}=\Gamma(n+1) / \Gamma\left(n+1-\alpha_{2}\right), v_{n}=\Gamma(n / q+1) / \Gamma(n / q-$ $\left.\alpha_{3}+1\right)$, and $\nu_{n}=\Gamma(n+1) / \Gamma\left(n+1-\alpha_{3}\right)$. 
Substituting (55) in (51), we have

$$
\begin{aligned}
& c_{1} \sum_{n=\left(N_{1}-1\right) q+1}^{\infty} a_{n} s_{n} t^{\left(n-s p_{1}\right) / q}+c_{2} \sum_{n=N_{2}}^{N_{1}-1} a_{n q} \rho_{n q} t^{n-\alpha_{2}} \\
& +c_{2} \sum_{n=\left(N_{1}-1\right) q+1}^{\infty} a_{n} r_{n} t^{\left(n-r p_{2}\right) / q}+c_{3} \sum_{n=N_{3}}^{N_{1}-1} a_{n q} v_{n q} t^{n-\alpha_{3}} \\
& +c_{3} \sum_{n=\left(N_{1}-1\right) q+1}^{\infty} a_{n} v_{n} t^{\left(n-v p_{3}\right) / q}=f\left(t, \sum_{n=0}^{\infty} a_{n} t^{n / q}\right) .
\end{aligned}
$$

Since the coefficients $a_{n q}, n=0, \ldots, N_{1}-1$, are known, we write the last equation in the form

$$
\begin{aligned}
& c_{1} \sum_{n=\left(N_{1}-1\right) q+1}^{\infty} a_{n} s_{n} t^{\left(n-s p_{1}\right) / q}+c_{2} \sum_{n=\left(N_{1}-1\right) q+1}^{\infty} a_{n} r_{n} t^{\left(n-r p_{2}\right) / q} \\
& \quad+c_{3} \sum_{n=\left(N_{1}-1\right) q+1}^{\infty} a_{n} v_{n} t^{\left(n-v p_{3}\right) / q} \\
& =f\left(t, \sum_{n=0}^{\infty} a_{n} t^{n / q}\right)-c_{2} \sum_{n=N_{2}}^{N_{1}-1} a_{n q} \rho_{n q} t^{n-\alpha_{2}} \\
& \quad-c_{3} \sum_{n=N_{3}}^{N_{1}-1} a_{n q} v_{n q} t^{n-\alpha_{3}} .
\end{aligned}
$$

Let $w=t^{1 / q}$; then $(57)$ reduces to

$$
\begin{aligned}
& \sum_{n=\left(N_{1}-1\right) q+1}^{\infty} c_{1} a_{n} s_{n} w^{n-s p_{1}}+\sum_{n=\left(N_{1}-1\right) q+1}^{\infty} c_{2} a_{n} r_{n} w^{n-r p_{2}} \\
& +\sum_{n=\left(N_{1}-1\right) q+1}^{\infty} c_{3} a_{n} v_{n} w^{n-v p_{3}} \\
& =f\left(w^{q}, \sum_{n=0}^{\infty} a_{n} w^{n}\right)-\sum_{n=N_{2}}^{N_{1}-1} c_{2} a_{n q} \rho_{n q} w^{q\left(n-\alpha_{2}\right)} \\
& \quad-\sum_{n=N_{3}}^{N_{1}-1} c_{3} a_{n q} v_{n q} w^{q\left(n-\alpha_{3}\right)} .
\end{aligned}
$$

Substituting $z=\left(N_{1}-1\right) q+1$ and

$$
\begin{aligned}
g(w)= & f\left(w^{q}, \sum_{n=0}^{\infty} a_{n} w^{n}\right)-\sum_{n=N_{2}}^{N_{1}-1} c_{2} a_{n q} \rho_{n q} w^{q\left(n-\alpha_{2}\right)} \\
& -\sum_{n=N_{3}}^{N_{1}-1} c_{3} a_{n q} \nu_{n q} w^{q\left(n-\alpha_{3}\right)}
\end{aligned}
$$

and shifting the indices to zero, we have

$$
\begin{gathered}
\sum_{n=0}^{\infty} c_{1} a_{n+z} s_{n+z} w^{n-s p_{1}+z}+\sum_{n=0}^{\infty} c_{2} a_{n+z} r_{n+z} w^{n-r p_{2}+z} \\
+\sum_{n=0}^{\infty} c_{3} a_{n+z} v_{n+z} w^{n-v p_{3}+z}=g(w) .
\end{gathered}
$$

To avoid singularity we multiply both sides in (60) by $w^{s p_{1}-z}$; we have

$$
\begin{aligned}
& \sum_{n=0}^{\infty} c_{1} a_{n+z} s_{n+z} w^{n}+\sum_{n=0}^{\infty} c_{2} a_{n+z} r_{n+z} w^{n-r p_{2}+s p_{1}} \\
& +\sum_{n=0}^{\infty} c_{3} a_{n+z} v_{n+z} w^{n-v p_{3}+s p_{1}}=w^{s p_{1}-z} g(w) .
\end{aligned}
$$

Equation (61) can be written as

$$
\begin{gathered}
\sum_{n=0}^{k_{1}-1} c_{1} a_{n+z} s_{n+z} w^{n}+\sum_{n=k_{1}}^{k_{2}-1}\left(c_{1} a_{n+z} s_{n+z}+c_{2} a_{n+z-k_{1}} r_{n+z-k_{1}}\right) \\
\cdot w^{n}+\sum_{n=k_{2}}^{\infty}\left(c_{1} a_{n+z} s_{n+z}+c_{2} a_{n+z-k_{1}} r_{n+z-k_{1}}\right. \\
\left.+c_{3} a_{n+z-k_{2}} v_{n+z-k_{2}}\right) w^{n}=w^{s p_{1}-z} g(w),
\end{gathered}
$$

where $k_{1}=s p_{1}-r p_{2}$ and $k_{2}=s p_{1}-v p_{3}$.

Applying analogs steps for deriving (22), we have

$$
\begin{gathered}
c_{1} a_{n+z} s_{n+z}+c_{2} a_{n+z-k_{1}} r_{n+z-k_{1}}+c_{3} a_{n+z-k_{2}} v_{n+z-k_{2}} \\
=\frac{1}{(n-j) !}\left[\frac{\mathrm{d}^{n-j}}{\mathrm{~d} w^{n-j}} g(w)\right]_{w=0} \quad \text { for } n \geq k_{2},
\end{gathered}
$$

where $j=s p_{1}-z$. From the last recursion we determine $a_{n+z}, n \geq k_{2}$.

Remark 5. Our derivation is based on the facts that the nonlinear function is smooth and the fractional differential equations is of constant coefficients. In case if one of these conditions does not hold, a modified treatment will be considered as we will see in Example 7.

\subsection{Numerical Results}

Example 6. Consider the Bagely-Torvik initial value problem

$$
\begin{aligned}
& D_{0^{+}}^{5 / 2} u(t)+D_{0^{+}}^{2} u(t)-2 \sqrt{\pi} D_{0^{+}}^{1 / 2} u(t)+4 u(t)=g(t), \\
& t \in[0,1], \\
& u(0)=u^{\prime}(0)=u^{\prime \prime}(0)=0,
\end{aligned}
$$

where

$$
g(t)=4 t^{9}-\frac{131072}{12155} t^{17 / 2}+72 t^{7}+\frac{49152}{143 \sqrt{\pi}} t^{13 / 2} .
$$

This example has been discussed in [21] using a Chebyshev spectral method, where the solution has been approximated by the shifted Chebyshev polynomials with different degrees. Then the exact solution $u(t)=t^{9}$ was obtained by considering the shifted Chebyshev polynomial of degree 9. We mention here that there are simple typos in presenting 
the example in (64) and in (66) and we correct them here. where Applying the current algorithm we have

$$
\begin{aligned}
p_{1} & =5, \\
p_{2} & =4, \\
p_{3} & =1, \\
q_{1} & =q_{2}=q_{3}=2, \\
q & =\operatorname{lcm}\left(q_{1}, q_{2}, q_{3}\right)=2, \\
s & =1, \\
r & =1, \\
v & =1, \\
k_{1} & =1, \\
k_{2} & =4 .
\end{aligned}
$$

We expand the solution in infinite series of the form $u(t)=$ $\sum_{n=0}^{\infty} a_{n} t^{n / 2}$. The initial condition in (65) yields $a_{0}=a_{2}=a_{4}=$ 0 . Let $t=w^{2}$; applying (59) we have

$$
\begin{aligned}
g(w)= & 4 w^{18}-\frac{131072}{12155} w^{17}+72 w^{14}+\frac{49152}{143 \sqrt{\pi}} w^{13} \\
& -4\left(\sum_{n=0}^{\infty} a_{n} w^{n}\right)
\end{aligned}
$$

and it holds that

$$
\begin{aligned}
& \left.\frac{\mathrm{d}^{m}}{\mathrm{~d} w^{m}} g(w)\right|_{w=0} \\
& \quad= \begin{cases}-4 a_{1} & \text { if } m=1 \\
-4 \times 2 ! a_{2} & \text { if } m=2 \\
\vdots & \vdots \\
13 !\left(\frac{49152}{143 \sqrt{\pi}}-4 a_{13}\right) & \text { if } m=13 \\
14 !\left(72-4 a_{14}\right) & \text { if } m=14 \\
-4 \times 15 ! a_{15} & \text { if } m=15 \\
17 !\left(-\frac{131072}{12155}-4 a_{17}\right) & \text { if } m=17 \\
18 !\left(4-4 a_{18}\right) & \text { if } m=16 \\
-4 ! a_{19} & \text { if } m=18 \\
\vdots & \text { if } m=19\end{cases}
\end{aligned}
$$

Using (63) and (69), we have

$$
\begin{gathered}
a_{n+5} s_{n+5}+a_{n+4} r_{n+4}-2 \sqrt{\pi} a_{n+1} v_{n+1} \\
=\left.\frac{1}{n !}\left(\frac{\mathrm{d}^{n}}{\mathrm{~d} w^{n}} g(w)\right)\right|_{w=0}, \quad n \geq 4,
\end{gathered}
$$

$$
\begin{aligned}
& s_{n+5}=\frac{\Gamma((n+5) / 2+1)}{\Gamma((n+5) / 2-3 / 2)}, \\
& r_{n+4}=\frac{(n+4)(n+2)}{4}, \\
& v_{n+1}=\frac{\Gamma((n+1) / 2+1)}{\Gamma((n+1) / 2+1 / 2)} .
\end{aligned}
$$

Since $g(w)$ is smooth, then $a_{n+5}=0$, for $n<4$. We now apply the last recursion together with $a_{0}=\cdots=a_{8}=0$, to compute $a_{n+5}$, for $n \geq 4$. For $n=4$, we have

$$
a_{9} s_{9}+a_{8} r_{8}-2 \sqrt{\pi} a_{5} v_{5}=g^{(4)}(0)=0,
$$

and thus $a_{9}=0$.

Applying analogous arguments yields $a_{10}=\cdots=a_{17}=0$. For $n=13$, we have

$$
a_{18} s_{18}+a_{17} r_{17}-2 \sqrt{\pi} a_{14} v_{14}=\frac{49152}{143 \sqrt{\pi}}-4 a_{13}
$$

which, together with $a_{17}=a_{14}=a_{13}=0$, implies that $a_{18}=1$. For $n=14$, we have

$$
a_{19} s_{19}+a_{18} r_{18}-2 \sqrt{\pi} a_{15} v_{15}=72-4 a_{14} \text {, }
$$

but $a_{18}=1$ and $a_{15}=a_{14}=0$; thus $a_{19}=0$. Applying the same steps yields $a_{20}=a_{21}=0$. For $n=17$, we have

$$
a_{22} s_{22}+a_{21} r_{21}-2 \sqrt{\pi} a_{18} v_{18}=-\frac{131072}{12155}-4 a_{17}
$$

and thus $a_{22}=0$.

Proceeding in the same manner, we have $a_{n+1}=0$ for $n \geq$ 18. Thus,

$$
u(t)=a_{18} t^{18 / 2}=t^{9}
$$

and the exact solution of problem (64)-(65) is obtained.

In the following example we show that the current algorithm can be applied to more general multiterm fractional differential equations which are not necessary of the form in (51).

Example 7. Consider the nonlinear three-term fractional initial value problem

$$
\begin{aligned}
& t D_{0^{+}}^{4 / 3} u(t)+D_{0^{+}}^{1 / 3} u(t)+t^{19 / 6} D_{0^{+}}^{1 / 6} u(t) \\
& \quad=\frac{33}{4 \Gamma(8 / 3)} t^{8 / 3}+\frac{1296}{935 \Gamma(5 / 6)} u^{2}(t)
\end{aligned}
$$

with

$$
u(0)=u^{\prime}(0)=0 \text {. }
$$

The exact solution for this problem is $u(t)=t^{3}$. 
Applying the current algorithm we have $q=6$. We then expand the solution in an infinite series of the form $u(t)=$ $\sum_{n=0}^{\infty} a_{n} t^{n / 6}$. From the initial condition (78), we have $a_{0}=$ $a_{6}=0$. To guarantee the existence of the fractional derivative $D_{0^{+}}^{4 / 3} u(t)$ we choose $a_{1}=\cdots=a_{5}=0$. Let $w=t^{1 / 6}$; then (77) reduces to

$$
\begin{aligned}
a_{1} r_{1} & +\sum_{n=0}^{18}\left(a_{n+2} s_{n+2}+a_{n+2} r_{n+2}\right) w^{n} \\
& +\sum_{n=19}^{\infty}\left(a_{n+2} s_{n+2}+a_{n+2} r_{n+2}+a_{n-18} v_{n-18}\right) w^{n} \\
= & \frac{33 w^{16}}{4 \Gamma(8 / 3)}+\frac{1296}{935 \Gamma(5 / 6)}\left(\sum_{n=0}^{\infty} a_{n} w^{n}\right)^{2},
\end{aligned}
$$

where $s_{n}=\Gamma(n / 6+1) / \Gamma(n / 6-1 / 3), r_{n}=\Gamma(n / 6+1) / \Gamma(n / 6+$ $2 / 3)$, and $v_{n}=\Gamma(n / 6+1) / \Gamma(n / 6+5 / 6)$.

Performing the $n$th derivatives one can see that $a_{n}=0$ for $n<16$. For $n=16$, we have

$$
a_{18} s_{18}+a_{18} r_{18}=\frac{33}{4 \Gamma(8 / 3)}
$$

which yields $a_{18}=1$.

Also, $a_{19}=\cdots=a_{35}=0$.

For $n=36$, we have

$$
a_{38} s_{38}+a_{38} r_{38}+a_{18} v_{18}=\frac{1296}{935 \Gamma(5 / 6)},
$$

which yields $a_{38}=0$. Thus

Following analogous steps, we have $a_{n}=0$ for $n>36$.

$$
u(t)=a_{18} t^{18 / 6}=t^{3}
$$

which is the exact solution.

\section{Conclusion}

For fractional differential equations of order $n-1<\alpha<$ $n$, it is common to obtain a series solution in the form $\sum_{n=0}^{\infty} a_{n} t^{\alpha(n)}$. The question is how to obtain the coefficients $a_{n}, n=0,1, \ldots$ Naturally, if the problem is of fractional order, the differentiation is also of fractional order. In this paper, we presented a new algorithm for obtaining a series solution for nonlinear multiterm fractional differential equations of Caputo type, where we overcome the use of fractional differentiation. We employed a transformation that allows us to use ordinary differentiation rather than fractional differentiation to recursively compute the coefficient of the series expansion. Then the terms of the series, $a_{n}$, are computed sequentially using a closed form formula. We applied the new algorithm to several types of multiterm fractional differential equations, where accurate solutions as well as exact solutions in closed forms have been obtained. For one example it is noted that the current algorithm is more efficient than the ADM as it is more easier to apply and it produces the exact solution while the ADM does not. We have developed the new algorithm for two- and three-term fractional equations, while the idea can be extended to multiterm fractional equations of arbitrary order; obtaining a general formula in this case is not an easy task. The current algorithm can be modified to deal with the fractional multiterm time-diffusion equations.

\section{Conflicts of Interest}

The authors declare that they have no conflicts of interest.

\section{Acknowledgments}

The authors gratefully acknowledge the support of the UAE University under Grant COS/IRG-11/15.

\section{References}

[1] M. Inc, "The approximate and exact solutions of the spaceand time-fractional Burgers equations with initial conditions by variational iteration method," Journal of Mathematical Analysis and Applications, vol. 345, no. 1, pp. 476-484, 2008.

[2] L. Song and H. Zhang, "Application of homotopy analysis method to fractional KdV-Burgers-KURamoto equation," Physics Letters A, vol. 367, no. 1-2, pp. 88-94, 2007.

[3] N. H. Sweilam, M. M. Khader, and R. F. Al-Bar, "Numerical studies for a multi-order fractional differential equation," Physics Letters A, vol. 371, no. 1-2, pp. 26-33, 2007.

[4] V. Daftardar-Gejji and S. Bhalekar, "Solving multi-term linear and non-linear diffusion-wave equations of fractional order by Adomian decomposition method," Applied Mathematics and Computation, vol. 202, no. 1, pp. 113-120, 2008.

[5] W. He, "Adomian decomposition method for fractional differential equations of Caputo Hadamard type," Journal of Computational Complexity and Applications, vol. 2, no. 4, pp. 160-162, 2016.

[6] H. Jafari and V. Daftardar-Gejji, "Solving linear and nonlinear fractional diffusion and wave equations by Adomian decomposition," Applied Mathematics and Computation, vol. 180, no. 2, pp. 488-497, 2006.

[7] S. Momani and K. Al-Khaled, "Numerical solutions for systems of fractional differential equations by the decomposition method," Applied Mathematics and Computation, vol. 162, no. 3, pp. 1351-1365, 2005.

[8] G. C. Wu, D. Baleanu, and H. P. Xie, "Riesz Riemann-Liouville difference on discrete domains," Chaos, vol. 26, no. 8, Article ID 084308, 2016.

[9] C. Yang and J. Hou, "An approximate solution of nonlinear fractional differential equation by laplace transform and adomian polynomials," Journal of Information and Computational Science, vol. 10, no. 1, pp. 213-222, 2013.

[10] A. Elsaid, M. S. Abdel Latif, and F. M. Kamal, "Series solution for fractional Riccati differential equation and its convergence," Journal of Fractional Calculus and Applications, vol. 6, no. 2, pp. 186-196, 2015.

[11] H. M. Srivastava, J. A. Tenreiro Machado, and X.-J. Yang, "Approximate methods for local fractional differential equations," in Fractional Dynamics, C. Cattani, H. M. Srivastava, and X.-J. Yang, Eds., pp. 244-258, Emerging Science Publishers (De Gruyter Open), Berlin, Germany, 2015. 
[12] X.-J. Yang, D. Baleanu, and H. M. Srivastava, Local Fractional Integral Transforms and Their Applications, Academic Press (Elsevier Science Publishers), Amsterdam, The Netherlands, 2016.

[13] M. Al-Refai, M. A. Hajji, and M. Syam, "An efficient series solution for fractional differential equations," Abstract and Applied Analysis, vol. 2014, Article ID 891837, 7 pages, 2014.

[14] M. K. Al-Srihin, Series solutions of multi-term fractional differential equations [M.S. thesis], United Arab Emirates University, Al Ain, United Arab Emirates, 2015.

[15] A. A. Kilbas, H. M. Srivastava, and J. J. Trujillo, Theory and Applications of Fractional Differential Equations, vol. 204 of North-Holland Mathematical Studies, Elsevier Science Publishers, Amsterdam, The Netherlands, 2006.

[16] I. Podlubny, Fractional Differential Equations. An Introduction to Fractional Derivatives, Fractional Differential Equations, Some Methods of Their Solution and Some of Their Applications, vol. 198 of Mathematics in Science and Engineering, Academic Press, San Diego, Calif, USA, 1999.

[17] G. Adomian, "A review of the decomposition method and some recent resuluts for nonlinear equations," Mathematical and Computer Modelling, vol. 13, no. 7, pp. 17-43, 1990.

[18] J.-S. Duan, "Convenient analytic recurrence algorithms for the Adomian polynomials," Applied Mathematics and Computation, vol. 217, no. 13, pp. 6337-6348, 2011.

[19] L.-L. Huang, G.-C. Wu, M. M. Rashidi, and W.-H. Luo, "Chaos analysis of the nonlinear duffing oscillators based on the new Adomian polynomials," Journal of Nonlinear Science and its Applications. JNSA, vol. 9, no. 4, pp. 1877-1881, 2016.

[20] M. Blasik, "Numerical scheme for a two-term sequential fractional differential equation," Scientific Research of the Institute of Mathematics and Computer Science, vol. 2, no. 10, pp. 17-29, 2011.

[21] E. H. Doha, A. H. Bhrawy, and S. S. Ezz-Eldien, "A Chebyshev spectral method based on operational matrix for initial and boundary value problems of fractional order," Computers \& Mathematics with Applications, vol. 62, no. 5, pp. 2364-2373, 2011. 




Advances in

Operations Research

vatem alat4

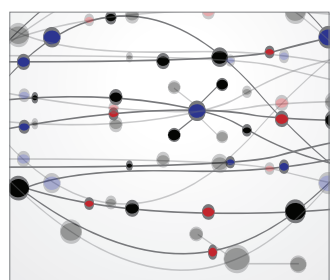

\section{The Scientific} World Journal
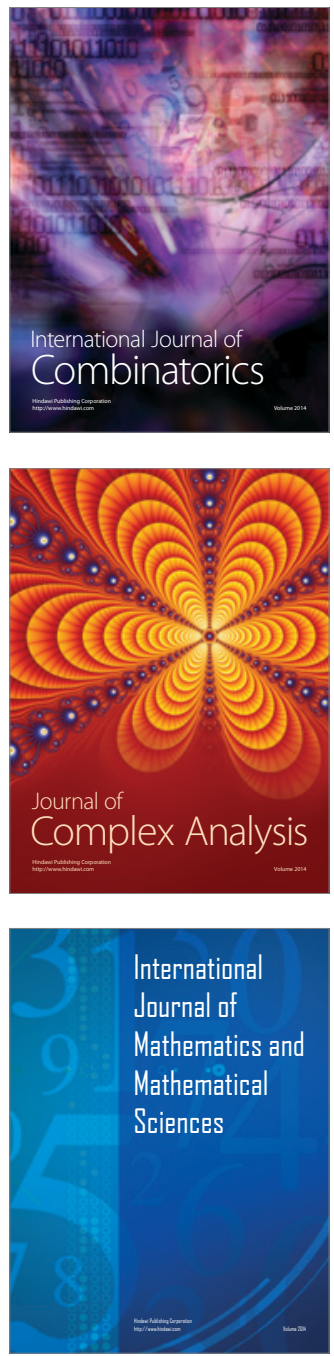
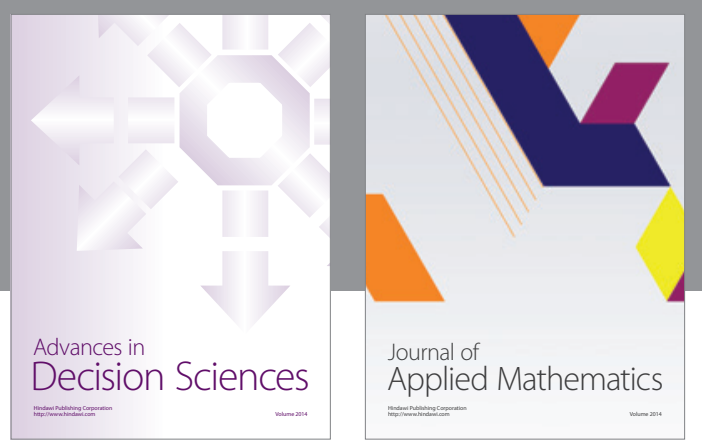

Algebra

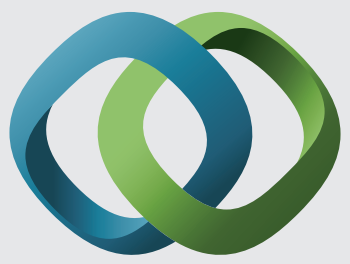

\section{Hindawi}

Submit your manuscripts at

https://www.hindawi.com
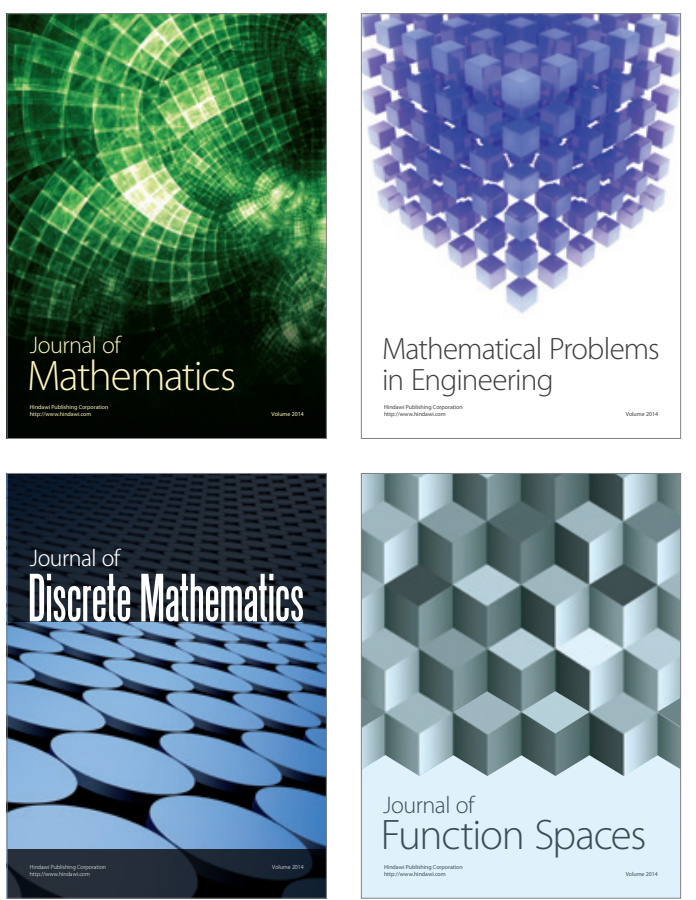

Mathematical Problems in Engineering
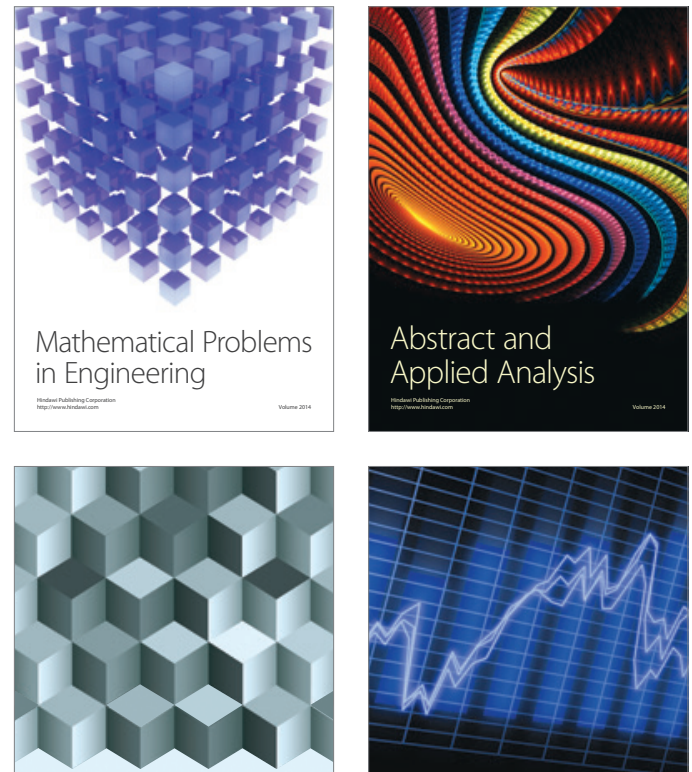

Journal of

Function Spaces



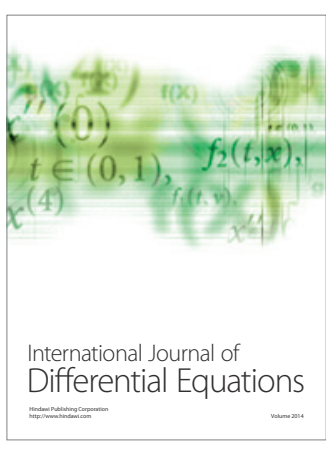
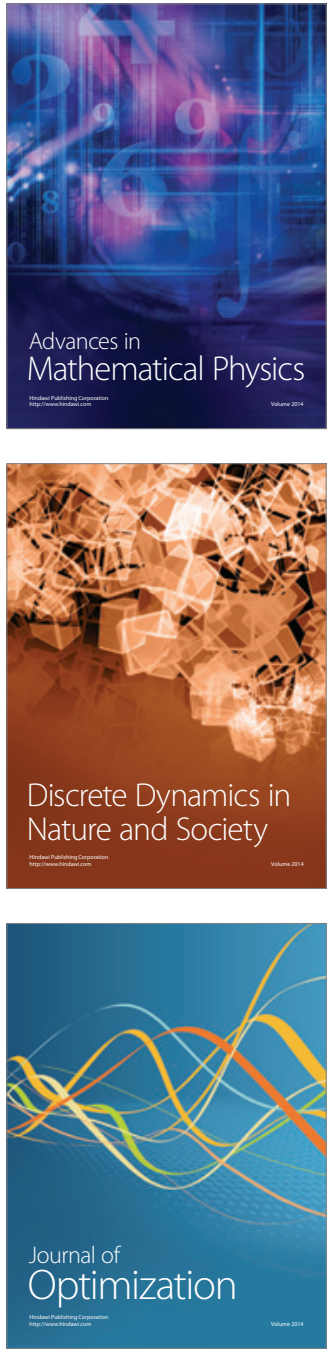